\section{Experiencia de conteo rápido durante el proceso electoral 2009}

Instituto Universitario de Opinión Pública

\section{Resumen}

Este artículo busca ofrecer una visión panorámica de lo que constituyó el primer esfuerzo sistemático de conteo rápido en $\mathrm{El}$ Salvador desarrollado por una amplia red de observación ciudadana a nivel nacional. Así, en primer lugar, se explica la metodología del conteo rápido. Luego se describe la fase preparatoria del proceso y las áreas bajo las cuales funcionó el dispositivo de observación. En un tercer apartado se expone el diseño muestral utilizado en ambas jornadas de observación. El cuarto apartado contiene los resultados pormenorizados de la observación de ambas jornadas electorales, contrastando los resultados nacionales con algunos de los estratos en los que se segmentó la muestra. En el quinto, se exponen las recomendaciones al sistema electoral derivadas de los informes de observación. Finalmente, se cierra con algunas valoraciones generales sobre el proceso.
Palabras clave: análisis, conteo rápido, democracia, elecciones, El Salvador, observación electoral, participación ciudadana. 
Los primeros antecedentes de conteos rápidos realizados en el marco de iniciativas ciudadanas de observación electoral nacional se remontan a las elecciones filipinas de 1986, en las que el Movimiento Nacional Ciudadano para Elecciones Libres (NAMFREL, por sus siglas en inglés) llevó a cabo una operación de recolección de datos en la mayoría de las mesas de votación que permitió evidenciar el fraude masivo que el régimen del presidente Ferdinand Marcos intentaba implementar (Estok, Nevitte y Cowan, 2002). Más tarde, en Chile, el Comité pro Elecciones Libres (CEL), con el apoyo del Instituto Nacional Demócrata (NDI, por sus siglas en inglés), desarrolló un conteo rápido durante el plebiscito de 1988, en el que la población rechazó la ampliación del período presidencial de Pinochet. En los años noventa e inicios de dos mil, cada vez más organizaciones cívicas de países con democracias emergentes han impulsado procesos de observación electoral doméstica, lo que ha dado lugar a un movimiento mundial de monitoreo ciudadano de los procesos electorales. En la actualidad, más de 70 países alrededor del mundo han impulsado procesos de observación ciudadana durante sus comicios, la mayoría de los cuales han contado con la asistencia técnica del NDI ${ }^{1}$, entidad pionera en el ámbito de la observación y transparencia electoral.

En Centroamérica, la organización Ética y Transparencia (ET) desarrolló, desde 1986, experiencias de observación electoral; y, más recientemente, en Guatemala, el grupo Mirador Electoral realizó un conteo rápido durante las elecciones presidenciales de 2007. En El Salvador, hasta antes de las elecciones de 2009, las experiencias de conteo rápido han sido las impulsadas por el Instituto Universitario de Opinión Pública (IUDOP), desde 1994. La modalidad de conteo rápido utilizada por el IUDOP, hasta las elecciones de 2006, se basó en la asignación de personal a diferentes centros de votación de algunos de los municipios más importantes del país, para que enviaran información de los resultados del escrutinio final en diferentes juntas receptoras de votos ${ }^{2}$.

Aunque esta metodología no estaba basada en una muestra probabilística, su utilización permitió monitorear, durante los procesos electorales postconflicto, los resultados electorales en algunos de los municipios más importantes del país y proveer al público, durante la misma noche de la jornada electoral, de información sobre las tendencias de los resultados electorales. La calidad de la jornada se exploraba a través de las encuestas de salida que se aplicaban en los mismos municipios donde se realizaba el conteo rápido, las cuales recogían la perspectiva ciudadana sobre el proceso electoral.

Adicionalmente a los esfuerzos del IUDOP, desde hace una década emergieron algunas iniciativas de observación nacional de la calidad de la jornada, que se desplegaron el día de la elección. Merece especial mención el esfuerzo realizado por la Procuraduría para la Defensa de los Derechos Humanos (PDDH), que durante varias elecciones ha desplegado grupos de observación ciudadana en diferentes municipios del país. Esta entidad ha emitido, habitualmente con posterioridad, informes sobre las irregularidades observadas y recomendaciones para mejorarlas, lo que ha contribuido a abrir la brecha en materia de observación electoral nacional. En el marco del proceso electoral 2009, la PDDH jugó un importante papel no solo en el monitoreo de las jornadas electorales, sino en la observación integral del proceso electoral. En repetidas oportunidades su titular se pronunció sobre violaciones al Código Electoral durante la campaña electoral, así como en torno a la inaplicabilidad de la ley por parte del máximo organismo electoral.

1. En Latinoamérica, los países que han impulsado experiencias de conteo rápido son Chile, Ecuador, Guatemala, Guyana, México, Nicaragua, Panamá, Perú y República Dominicana. Otros países alrededor del mundo son Albania, Angola, Bangladés, Bielorrusia, Bulgaria, Burundi, Camboya, Croacia, Eslovaquia, Georgia, Ghana, Indonesia, Kenia, Kosovo, Malaui, Montenegro, Yugoslavia, Rumania, Ucrania y Zambia.

2. Durante las elecciones legislativas y municipales de 2006, el IUDOP realizó un conteo rápido en cuatro municipios del país: San Salvador y Santa Tecla en el Área Metropolitana de San Salvador, San Miguel en la zona oriental y Santa Ana en la región occidental. 
A inicios de 2008, el NDI sostuvo conversaciones con la Universidad Centroamericana "José Simeón Cañas" (UCA) para explorar la posibilidad de que el IUDOP realizara un esfuerzo de observación electoral nacional, bajo la metodología de conteo rápido utilizada por el NDI, la cual se basa en la observación sistemática de la jornada electoral en una muestra probabilística de juntas receptoras de votos. Luego de varias reuniones con los equipos de asesoría internacional del NDI, en las que se dio a conocer la metodología utilizada, las experiencias en otros países y se analizó el contexto político y las condiciones institucionales para impulsar un proyecto de esta naturaleza en El Salvador, el IUDOP asumió el desafío de impulsar un esfuerzo de monitoreo electoral de los procesos electorales de 2009, con el apoyo técnico del NDI. En este marco, el proyecto tuvo como propósito principal contribuir a la promoción de una mayor transparencia y confianza en un contexto institucional de elevada desconfianza ciudadana hacia el proceso y los organismos de gestión electoral ${ }^{3}$. El presente artículo busca ofrecer una visión más amplia de lo que constituyó el primer esfuerzo sistemático de conteo rápido desarrollado por una amplia red de observación ciudadana a nivel nacional. Una primera parte de este trabajo se centra en explicar la metodología del conteo rápido. Un segundo capítulo describe la fase preparatoria del proceso y las áreas bajo las cuales funcionó el dispositivo de observación. En un tercer apartado se expone el diseño muestral utilizado en ambas jornadas de observación. Un cuarto capítulo contiene los resultados pormenorizados de la observación de ambas jornadas electorales, contrastando los resultados nacionales con algunos de los estratos en los que se segmentó la muestra, tomando como base los informes que se generaron en ambos eventos electorales. Un quinto apartado expone las recomendaciones al sistema electoral derivadas de los informes de observación. Y un acápite final contiene algunas valoraciones generales sobre el proceso.

\section{El proceso del conteo rápido}

El conteo rápido es un valioso método para monitorear elecciones, que se realiza mediante la observación de lo que ocurre el día de la elección en una muestra representativa de mesas electorales, a partir del cual se hacen estimaciones estadísticas sobre los resultados electorales y se evalúa la calidad global de la jornada electoral. A diferencia de otras metodologías de observación, la unidad de análisis en este tipo de conteo no es el centro de votación, sino la mesa de votación o junta receptora de votos (JRV), por lo que la selección de la muestra se hace sobre la base del listado total de mesas electorales que el organismo electoral instalará a nivel nacional en una elección. Con el fin de garantizar que la muestra sea representativa del total de mesas electorales, el conteo rápido se basa en muestras grandes, seleccionadas de forma probabilística. Esto permite hacer estimaciones estadísticas confiables sobre el comportamiento electoral de toda la población.

Bajo esta metodología, se asignan observadores a centros de votación y JRV específicas, quienes registran información clave en formularios estandarizados sobre la calidad de la jornada electoral y los resultados electorales. Estos mismos voluntarios transmiten esta información, en diferentes momentos de la jornada, a un centro de recolección de datos, donde se consolida y analiza. Esta transmisión se desarrolla en dos momentos: (a) en la mañana: sobre el proceso de instalación de la JRV y acerca del inicio de la votación. Los voluntarios y voluntarias realizan la transmisión una vez inicia la votación. Y (b) en la tarde/noche: sobre el desarrollo de la votación y acerca del cierre de la votación y el escrutinio. Los voluntarios y voluntarias hacen la transmisión

3. Una encuesta sobre preferencias electorales cursada por el IUDOP en septiembre de 2008 reveló que el $63.8 \%$ de los salvadoreños tenía poca o ninguna confianza en el TSE; esta proporción se incrementó a $73.4 \%$ en la encuesta de evaluación de año y sobre las elecciones legislativas y municipales de 2009 , cursada en noviembre de 2008. 
luego de haberse firmado el acta de cierre del escrutinio final en la JRV.

Para ello, un componente sustancial de la observación es la red de voluntarios y voluntarias que participan monitoreando la jornada electoral con el fin de salvaguardar el respeto a la voluntad popular. A este respecto, un valor agregado de esta metodología es estimular la participación ciudadana en los asuntos electorales, pues permite que los propios ciudadanos se involucren en la vigilancia de sus procesos electorales.

El mecanismo de transmisión de la información utilizado por los miembros de la red que observan la mesa electoral puede variar en función de la extensión geográfica de los países, así como de la tecnología y redes de comunicación disponibles. En el caso salvadoreño, la información fue transmitida a través de telefonía celular a una central telefónica ubicada en un centro de procesamiento de datos. Adicionalmente, con el fin de no depender de un solo flujo de información, que pudiese ser afectado por problemas técnicos o por bloqueos del sistema de comunicación, se generó un respaldo de la información recabada a través de la misma red de observadores. Para ello, el observador realizó una segunda llamada al coordinador de la red de su municipio y una tercera llamada a un grupo de ciudadanos que operaron desde sus hogares, a quienes transmitió exactamente la misma información que a la central de datos.

A diferencia de otras metodologías de conteo, que se centran en consignar únicamente los resultados de la votación, este proceso examina aspectos cualitativos de la jornada electoral, lo que permite hacer una evaluación de la calidad de la misma. De esta forma no solo se tiene una estimación de los resultados electorales, sino un análisis sistemático de los problemas o irregularidades suscitadas durante el día de la elección, a partir de lo cual se puede establecer el grado de confianza de los resultados electorales. Otra de las características de esta metodología es que la estimación de los resultados electorales se basa en los datos de las propias actas del escrutinio final, por lo que se fundamenta en datos objetivos y oficiales. Sin embargo, tiene la limitante de que se trata de una verificación de lo ocurrido el día de la jornada, por lo que un proceso de observación como este no siempre puede dar cuenta de manipulaciones previas al día de la elección, como la compra de votos o la suplantación de votantes en el padrón, que puedan afectar los resultados electorales.

Aunque el conteo rápido no es el único método para observar integralmente una elección, ha sido una importante vía para determinar el grado de confianza de los resultados electorales. Un conteo rápido puede jugar un papel central en el desarrollo de elecciones sumamente competitivas o tensas, disuadiendo intentos de fraude o, en otros casos, previniendo conflictos sociales en escenarios donde la población desconfía de los resultados electorales.

\section{La fase preparatoria del conteo rápido}

El desarrollo de un proyecto de observación nacional es una empresa grande y compleja, por lo que se requiere de un proceso preparatorio durante el semestre previo al proceso electoral. Para ello, se procedió a planificar paso a paso cada una de las etapas necesarias para el establecimiento de las capacidades técnicas, instaladas y de articulación territorial del proyecto. Una de las decisiones más importantes que se tomaron en esta etapa del proceso fue la relativa al tamaño de la muestra. Para las elecciones legislativas y municipales del 18 de enero, se decidió observar a nivel nacional un total de $1,500 \mathrm{JRV}$; y para la elección presidencial del 15 de marzo, una muestra de 2,500 JRV. La decisión de aumentar en 1,000 JRV la muestra de la elección presidencial respondió a la necesidad de reducir el error muestral y, con ello, aumentar la precisión en la estimación de los resultados electorales.

Para facilitar la implementación del proyecto, el equipo del IUDOP se reorganizó en áreas de trabajo, a fin de impulsar la serie de actividades que debían gestarse paralelamente, en razón de la lógica interdependiente del proceso y la cercanía de las fechas de la elección. Las áreas de trabajo que se conformaron durante la fase preparatoria fueron dirección-coordinación, red de observadores, administración, 
logística, análisis, procesamiento y comunicaciones. A efectos de ilustrar la fase preparatoria, se reseñarán algunos de los principales procesos que se articularon en función del proyecto.

\subsection{Coordinación y gestión política}

El proyecto de observación demandó no solo una articulada coordinación interna, sino el establecimiento de una estrategia de acercamiento y coordinación con los órganos de gestión electoral, actores políticos, cuerpo diplomático, representantes de la cooperación internacional acreditados en el país y miembros de las principales misiones de observación internacional.

Uno de los aspectos cruciales para el avance del proceso es establecer un acuerdo con el máximo organismo electoral que acredite el trabajo de la red de observación. La experiencia en otros países da cuenta de que con frecuencia las autoridades electorales ven con desconfianza los esfuerzos de observación realizados por organizaciones nacionales, por lo que se resisten a la realización de conteos independientes de votos. No obstante, es fundamental que las organizaciones interesadas en hacer una observación doméstica traten de forjar una relación de cooperación con los organismos electorales, por varias razones. En primera instancia, porque en muchos de los países, incluyendo El Salvador, cualquier persona que quiere hacer labor de observación durante las elecciones requiere de una credencial oficial para poder permanecer en un centro de votación ${ }^{4}$; en segundo lugar, porque son las autoridades electorales las que cuentan con información relevante, como la lista completa de los centros de votación y la ubicación de las JRV, a partir de la cual se diseña la muestra.

Dado que el proyecto anticipó un despliegue importante de observadores y observadoras en las juntas receptoras de votos, quienes debían contar con información precisa sobre el desarrollo de toda la jornada electoral, desde la instalación de las JRV hasta el cierre y escrutinio final, era inviable realizar un proceso de observación sin contar con un acuerdo formal con el TSE para acreditar al contingente de observadores y observadoras. Esto supuso una negociación con el TSE, que concluyó con un convenio de cooperación con el IUDOP y el NDI. En él se estableció el marco de trabajo conjunto que garantizaría que la red de observadores contaría con las credenciales para poder observar sin dificultad la jornada electoral. A su vez, el TSE emitió el Reglamento General para la Observación Electoral en El Salvador, en el que se reconoció formalmente la figura de observador nacional y se reguló su participación en los comicios. Para hacer avanzar con mayor celeridad este proceso, fue importante el respaldo político que otorgaron al proyecto algunas embajadas y organismos internacionales acreditados en el país.

Con la finalidad de mantener informada a la comunidad internacional de los avances del proyecto, se sostuvieron reuniones grupales y bilaterales con algunos embajadores acreditados en el país, representantes de agencias internacionales, de la Misión de Observación Electoral de la Organización de Estados Americanos (MOE-OEA) y de la Misión de Observación Electoral de la Unión Europea (MOE-UE).

\subsection{Diseño muestral}

Una de las principales fortalezas del conteo es que el diseño de la metodología de recolección y análisis de datos se basa en principios de estadística ampliamente aceptados. Esto permite que los organizadores puedan señalar con autoridad los resultados electorales (Estok, Nevitte y Cowan, 2002). De ahí la importancia de que los datos que se recaben tengan la solidez y confiabilidad necesaria, y esto depende en buena medida del diseño de la muestra. En ese contexto, una actividad de gran importancia fue el diseño de las muestras probabilísticas

4. Aunque no hay ningún artículo en el Código Electoral que prohíba expresamente la presencia de ciudadanos observando la jornada electoral, tampoco hay ninguna disposición legal que lo permita, por lo que, regularmente, ciudadanos nacionales o extranjeros que han participado en esfuerzos de observación han requerido la emisión de una credencial oficial por parte del máximo organismo electoral. 